\title{
Use of Intralesional Bleomycin for Oral Hemangioma of Sturge-Weber Syndrome
}

\author{
${ }^{1}$ Devayani Shinde, ${ }^{2}$ Yogesh G Dabholkar, ${ }^{3}$ Akanksha A Saberwal, ${ }^{4}$ Haritosh Kamalakar Velankar, ${ }^{5}$ Adip K Shetty
}

\begin{abstract}
Sturge-Weber syndrome (SWS) or encephalotrigeminal angiomatosis is a rare neurocutaneous disorder characterized with vascular malformations and capillary venous angiomas involving the face, choroid of the eye and leptomeninges with port wine stain, seizures activity and mental retardation. In this paper we report a case of a patient affected by Sturge-Weber syndrome presenting with tongue hemangioma and emphasize the importance of an accurate diagnosis and management in the clinical practice.
\end{abstract}

Keywords: Sturge-Weber, Neurocutaneous disorder, Hemangioma, Propranolol, Corticosteroids, Bleomycin.

How to cite this article: Shinde D, Dabholkar YG, Saberwal AA, Velankar HK, Shetty AK. Use of Intralesional Bleomycin for Oral Hemangioma of Sturge-Weber Syndrome. Int J Head Neck Surg 2015;6(1):17-19.

Source of support: Nil

Conflict of interest: None

\section{INTRODUCTION}

Sturge-Weber syndrome (SWS) is a congenital neurocutaneous syndrome characterized by unilateral facial cutaneous vascular malformation (nevus flammeus or port-wine stain) in association with ipsilateral leptomeningeal angiomatosis. It is a rare disorder that occurs sporadically with a frequency of 1:50,000. First described by Schirmer and later more specifically by Sturge in 1879 , it is also known as Sturge-Weber disease, craniofacial angiomatosis, encephalotrigeminal angiomatosis, etc. Oral manifestations of SWS are present in approximately 38\% of the cases, and they may involve hemangiomatous lesion in the lip, oral mucosa, gum, tongue and palatal region. ${ }^{1}$

\section{CASE REPORT}

A 13-year-old male child presented to our hospital with a swelling over the right side of tongue since 7 months of

\footnotetext{
${ }^{1,3}$ Resident, ${ }^{2}$ Professor, ${ }^{4}$ Professor and Head

${ }^{5}$ Assistant Professor

1-3,5 Department of ENT, Dr DY Patil Hospital and Research Centre Navi Mumbai, Maharashtra, India

${ }^{4}$ Department of ENT, Velankar Hospital, Mumbai, Maharashtra India

Corresponding Author: Haritosh Kamalakar Velankar Professor and Head, Department of ENT, Velankar Hospital Mumbai, Maharashtra, India, Phone: 02225283314, e-mail: drharitosh@yahoo.co.in
}

age (Fig. 1). The swelling was progressively increasing in size, painless, soft in consistency, causing no significant interference while speaking, chewing or swallowing. Further physical examination revealed conjunctival hemangioma in right eye, portwine stain on the right vestibule and microcephaly with a head circumference of $48 \mathrm{~cm}$. Patient gave a history of a submandibular hemangioma excision done at 7 years of age. Patient also reported a history of seizures at 6 months of age for which he received antiepileptic treatment for 2 years. Subsequently, there were no further episodes of seizures. But, the caretaker reported poor scholastic performance.

Routine hemogram and biochemical tests revealed no significant abnormalities. An ultrasound examination showed a vascular lesion in right side of tongue measuring $22 \times 20 \mathrm{~mm}$ suggestive of hemangioma with moderate vascularity. EEG was unremarkable. Contrast enhanced MRI with MR angiography reported an abnormal well-defined enhancing lesion in right lateral margin of tongue $2.5 \times 2.5 \times 1.5 \mathrm{~cm}$ suggestive of venolymphatic malformation (Fig. 2). Similar malformations were noted in the submandibular and posterior cervical region and right masseter muscle and gliosis with hemorrhagic residue in right frontotemporal perisylvian region with multiple abnormal dural and pial draining veins suggestive of arteriovenous fistulae (Fig. 3).

A diagnosis of SWS was finally made based on the history of seizure, learning disability and examination findings of portwine stain and intracranial arteriovenous fistulae.

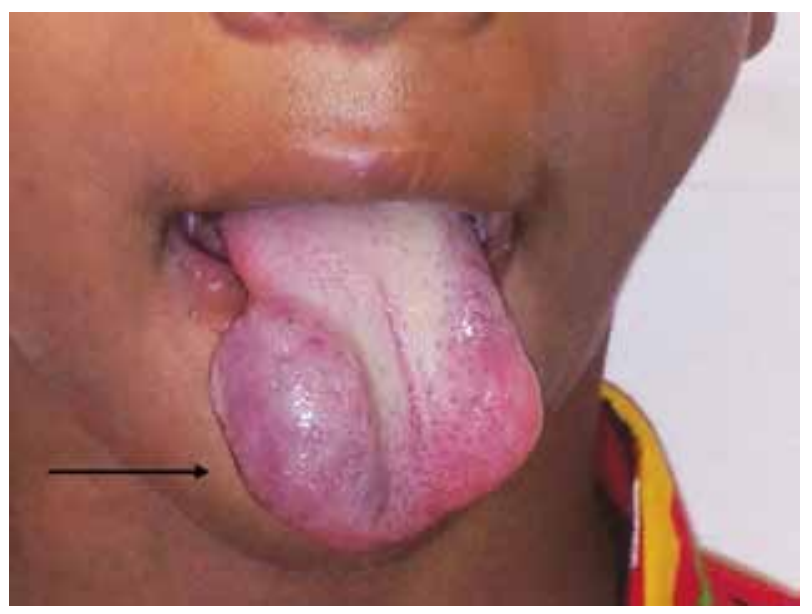

Fig. 1: Case of Sturge-Weber syndrome with a tongue hemangioma 
Patient was initially started on oral propranolol. However, after an attack of bronchospasm, this modality was abandoned. Patient was subsequently given a course of oral corticosteroids, but he developed raised intraocular pressure and the corticosteroids were tapered and stopped. A digital subtraction angiography (DSA) was performed to evaluate the type of flow in hemangioma which showed slow flow arteriovenous malformation in right side of tongue. A decision was made to treat the hemangioma with sclerotherapy. Initially, polidocanol and lipiodol solution was used for intralesional injections. ${ }^{2}$ However, no improvement was seen with this sclerosing agent. Subsequently, bleomycin was used as injection sclerotherapy at a dose of $0.5 \mathrm{mg} / \mathrm{kg}$ body weight using the two-needle technique (Fig. 4). This involved insertion of two needles in the lesion; the first needle was used to introduce the sclerosant, while the second needle drained the excess sclerosant, thereby minimizing extravasation of agent and subsequent risks. ${ }^{3}$ The lesion showed

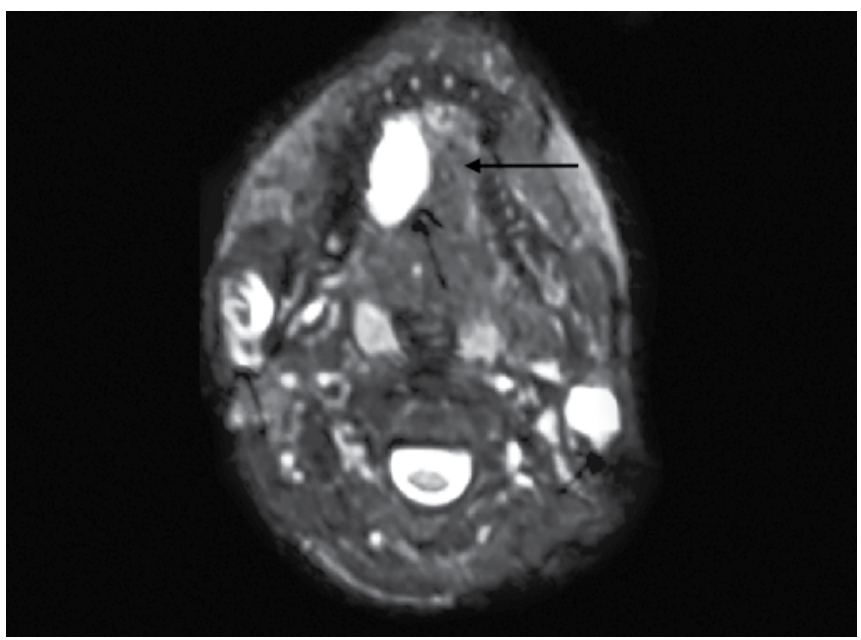

Fig. 2: Contrast MRI image of venolymphatic malformation in the right lateral margin of tongue

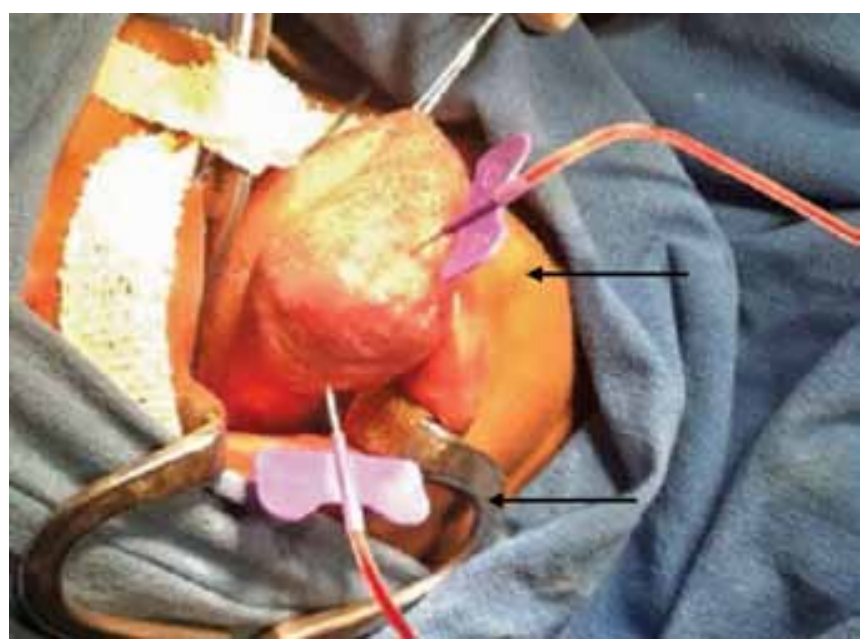

Fig. 4: Intraoperative image using two needle technique for sclerotherapy remarkable regression with a single dose at 2 months follow-up (Fig. 5).

\section{DISCUSSION}

Sturge-Weber syndrome is a rare neurocutaneous disorder. It is believed that it is caused by the abnormal persistence of an embryonal vascular system localized around the cephalic region of the neural tube. ${ }^{4}$

The SWS presents with neurological, cutaneous, ocular and oral manifestations that may or may not be associated with one another; however, the most evident clinical manifestation is the presence of the nevus flammeus or port-wine stain on the face. The ipsilateral leptomeningeal angiomatosis also represents one of the main signs of the syndrome. The diagnosis is based on the presence of nevus flammeus in the face, and association between convulsion and the nevus, irrespectively of the neurological loss, in addition to one of the following manifestations viz contralateral hemiparesis and

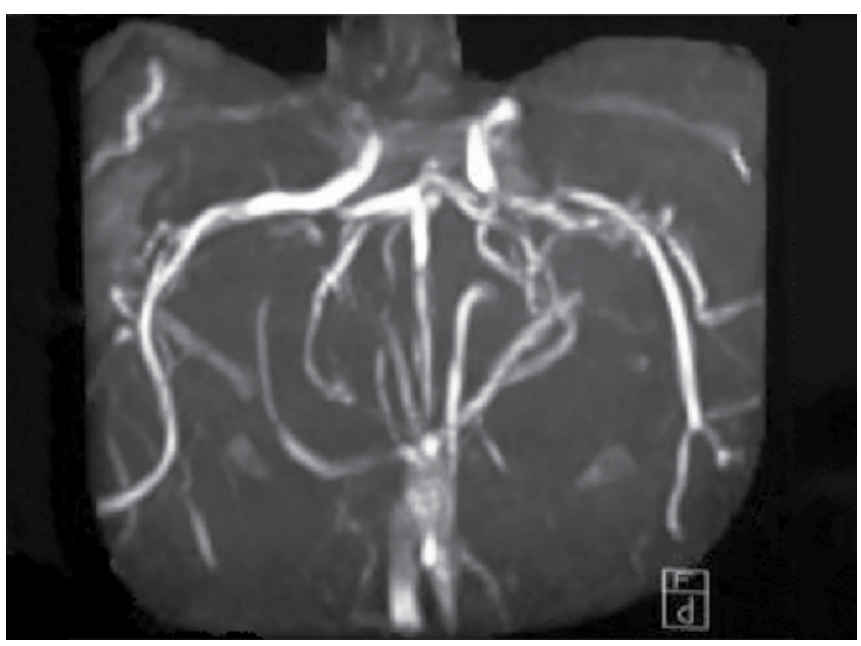

Fig. 3: MR angiography image showing multiple arteriovenous fistulae

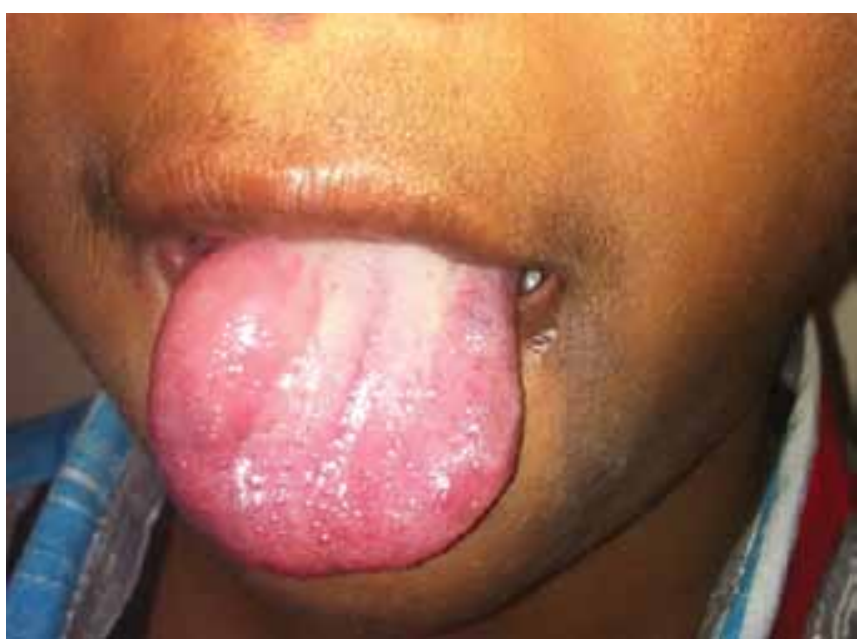

Fig. 5: Postsclerotherapy image of tongue hemangioma 
hemiatrophy, mental retardation and glaucoma. CT and MRI scans show diagnostic intracranial calcification. DSA can be helpful to provide type of flow. ${ }^{1}$

Though SWS is a rare condition, it should be considered as a differential diagnosis whenever any patient presents with vascular malformations. Our patient had presented with only a tongue hemangioma. A detailed history and examination coupled with a high index of clinical suspicion and relevant investigations were crucial in successfully diagnosing him as a SWS. The parents of all the diagnosed patients must receive counselling and should be educated about the potential complications of the disease as well.

Hemangiomas, although often small at birth, enter a proliferative phase in which growth may be rapid and unpredictable. Furthermore, involution often takes many years, with attendant psychological sequelae to the child. This is aggravated by the fact that most hemangiomas affect the head and neck and are visible and difficult to conceal.

Oral propranolol ( $2 \mathrm{mg} / \mathrm{kg}$ per day) is considered a well-tolerated and effective treatment modality for hemangiomas with mild adverse effects and low recurrence rates. ${ }^{5}$ Our patient was started on propranolol according to the recommended dosage. However, this procedure was abandoned in lieu of bronchospasm. Studies by Graaf et al reported potentially harmful adverse effects, including hypoglycemia, bronchospasm and hypotension despite propranolol being an effective treatment option for hemangiomas. ${ }^{6}$

Treatment of hemangiomas in infants with high doses of oral steroids $(5 \mathrm{mg} / \mathrm{kg} /$ day of prednisone for 6 to 8 weeks) has also been reported to be a safe, effective and rapid treatment modality with a response rate of up to $84 \%$. However, occult adverse effects like hypothalamicpituitary-adrenal axis suppression, hypertension, etc. usually go unnoticed without specific monitoring. ${ }^{7}$

Surgical excision can be curative, but surgeons often resort to performing incomplete excisions to avoid loss of muscle and nerve function for a benign condition. Also, the recurrence rate is high after incomplete excision and tumor remnants can grow aggressively causing symptoms worse than before surgery. ${ }^{8}$

Sclerotherapy with liquid denaturating agents has been an alternative to surgical excision of hemangiomas since the 1930. Sclerotherapy with polidocanol has shown excellent long-term results, especially in hemangiomas localized in the face. ${ }^{3}$ Complications of sclerotherapy include extravasation of sclerosant outside the hemangioma causing tissue necrosis or nerve damage, necrosis and ulceration for superficial lesions, deep vein thrombosis and/or arterial thrombosis. ${ }^{8}$ Bleomycin, an antineoplastic agent and OK-432 are currently the most popular sclerosants. Studies using bleomycin have produced promising results with up to $88 \%$ of significant tumor regression of which 36 to $63 \%$ had complete regression. No serious complications were seen with intralesional bleomycin therapy. The primary concern with bleomycin is a risk of pulmonary fibrosis. However, as the dosage used in sclerotherapy are much lower than those used in oncology, pulmonary fibrosis is uncommon with intralesional bleomycin. ${ }^{9}$

\section{CONCLUSION}

Sturge-Weber syndrome is a rare neurocutaneous disorder and a high index of clinical suspicion is required to reach this diagnosis. Various treatment modalities exist for treatment of hemangiomas including oral propranolol or corticosteroids as well as surgery. We recommend using bleomycin as intralesional sclerosing agent as an effective treatment for oral hemangioma.

\section{REFERENCES}

1. Neto FXP, Junior MAV, Ximenes LS, Jacob CCS, Junior AGR, Palheta CP, et al. Clinical features of Sturge-Weber syndrome. Int Arch Otorhinolaryngol 2008;12(4):565-570.

2. Winter H, Dräger E, Sterry W. Sclerotherapy for treatment of hemangiomas. Dermatol Surg 2000;26(2):105-108.

3. Puig S, Aref H, Brunelle F. Double-needle sclerotherapy of lymphangiomas and venous angiomas in children: a simple technique to prevent complications. Am J Roentgenol 2003 May;180(5):1399-1401.

4. Aydin A, Çakmakçi H, Kovanlikaya A, Dirik E. Sturge-Weber syndrome without facial nevus. 3. Pediatr Neurol 2000 May; 22(5):400-402.

5. Xiao Q, Li Q, Zhang B, Yu W. Propranolol therapy of infantile hemangiomas: efficacy, adverse effects, and recurrence. Pediatr Surg Int 2013 Jun;29(6):575-578.

6. de Graaf M, Breur JM, Raphaël MF, Vos M, Breugem CC, Pasmans SG. Adverse effects of propranolol when used in the treatment of hemangiomas: a case series of 28 infants. J Am Acad Dermatol 2011 Aug;65(2):320-327.

7. Bennett ML, Fleischer $\mathrm{AB} \mathrm{Jr}$, et al. Oral corticosteroid use is effective for cutaneous hemangiomas an evidence-based evaluation. Arch Dermatol 2001;137(9):1208-1221.

8. Crawford EA, Slotcavage RL, et al. Ethanol sclerotherapy reduces pain in symptomatic musculoskeletal hemangiomas. Clin Orthop Relat Res 2009 Nov;467(11):2955-2961.

9. Rozman Z, Thambidorai RR, Zaleha AM, et al. Lymphangioma: is intralesional bleomycin sclerotherapy effective? Biomed Imaging Interv J 2011 Jul-Sep;7(3):e18. 